\author{
ANTIPROTON YIELDS FOR STOCHASTIC ACCUMULATION \\ Carlos Hojvat and A. Van Ginneken \\ July 15,1981 \\ (Revised)
}

Two sets of parameters have been developed to study stochastic accumulation (See Notes on Stochastic Cooling Meeting of la July 1981). The question arises of the size of the transverse emittance required to achieve an antiproton yield of $1.2 \times 10^{8} \overline{\mathrm{p}} \mathrm{s} / \mathrm{MR}$ cycle for $4.5 \mathrm{GeV}$ and $8 \mathrm{GeV} \overline{\mathrm{p}}$ kinetic energies, given a full momentum acceptance of $4 \%$.

The results quoted below are obtained with a Monte Carlo program which uses a fit to the $\bar{p}$ production invariant cross section that has been presented previously. An article describing the program is in preparation.

If the $1.2 \times 10^{8}$ antiprotons arise from $2 \times 10^{12}$ protons on target then the required yields are;

1) $4.5 \mathrm{GeV}(5.4 \mathrm{GeV} / \mathrm{c})$ :

$$
y=\frac{1.2 \times 10^{8}}{2 \times 10^{12} \times 5.4 \times 0.04}=2.78 \times 10^{-4}\left(\frac{\text { antiprotons }}{\text { protons.GeV } / \cdot c}\right.
$$

2) $8.0 \mathrm{GeV}(8.9 \mathrm{GeV} / \mathrm{c})$ :

$$
y=\frac{1.2 \times 10^{8}}{2 \times 10^{12} \times 8.9 \times 0.04}=1.69 \times 10^{-4}\left(\frac{\text { antiprotons }}{\text { protons.GeV } / t}\right.
$$

To collect the antiprotons a lithium lens of $1 \mathrm{~cm}$ radius and a gradient of $1000 \mathrm{~T} / \mathrm{M}$ is assumed. For practical considerations we 
$\bar{p} \mathrm{p}$ Note 139

Page 2

will maintain the distance between the target center and the upstream end of the lens to be $14.5 \mathrm{~cm}$ for both antiproton energies. This requirement results in a $10 \mathrm{~cm}$ long lens for the $4.5 \mathrm{GeV}$ case and a $15 \mathrm{~cm}$ long one for the $8.0 \mathrm{GeV}$ case.

Consistent with previous calculations of energy deposited on the target we utilize a proton beam spot of $\sigma_{x}=\sigma_{y}=0.022 \mathrm{~cm}$. The target length is chosen to be $5 \mathrm{~cm}$ long Tungsten.

\section{$8.0 \mathrm{GeV}$ Antiprotons}

For $100 \mathrm{GeV}$ incident protons the required transverse emittance is $21 \pi \times 10^{-6} \mathrm{~m}$, with $17 \%$ of the antiprotons due to production by secondaries.

For $200 \mathrm{GeV}$ incident protons the required transverse emittance is $12 \pi \times 10^{-6} \mathrm{~m}$, with $25 \%$ of the antiprotons arising from secondary production.

\section{$4.5 \mathrm{GeV}$ Antiprotons}

The required yield is difficult to obtain with $100 \mathrm{GeV}$ protons. For example, for an acceptance of $40 \pi \times 10^{-6}$ we obtain a yield of $2.36 \times 10^{-4}$, or 1.18 times below the desired value. Secondaries contribute $28 \%$ of the total antiproton yield.

For $200 \mathrm{GeV}$ incident protons a transverse emittance of the order of $27 \pi \times 10^{-6} \mathrm{~m}$ is required, with $38 \%$ of the antiprotons due to secondary production.

\section{Conclusions}

The transverse emittances assumed for the Stochastic Accumulator design are approximately correct, depending on what proton energy is chosen.

It should be remembered that the minimum achievable cycle times are expected to be 1.68 seconds at $100 \mathrm{GeV}$ and 2.61 seconds at 200 $\mathrm{GeV}$. 